\title{
The structural neural substrates of persistent negative symptoms in first-episode of non-affective psychosis: voxel-based morphometry study
}

\author{
Audrey Benoit ${ }^{1,2}$, Michael Bodnar ${ }^{1,2,3}$, Ashok K. Malla ${ }^{2,3,4}$, Ridha Joober ${ }^{2,4}$ and Martin Lepage $e^{1,2,3,4 *}$ \\ Brain Imaging Group, Douglas Mental Health University Institute, Verdun, QC, Canada \\ 2 Prevention and Early Intervention Program for Psychoses, Douglas Mental Health University Institute, Verdun, OC, Canada \\ ${ }^{3}$ Department of Neurology and Neurosurgery, McGill University, Montreal, QC, Canada \\ ${ }^{4}$ Department of Psychiatry, McGill University, Montreal, OC, Canada
}

\section{Edited by:}

Zafiris J. Daskalakis, University of

Toronto, Canada

\section{Reviewed by:}

Paul Croarkin, Mayo Clinic, USA

Daniel Blumberger, Centre for

Addiction and Mental Health, Canada

${ }^{*}$ Correspondence:

Martin Lepage, Prevention and Early Intervention Program for Psychoses, Douglas Mental Health University Institute, Frank B. Common Pavilion, 6875 LaSalle Blvd., Verdun, OC,

Canada $\mathrm{H} 4 \mathrm{H} 1 \mathrm{R} 3$.

e-mail: martin.lepage@mcgill.ca

\begin{abstract}
Objectives: An important subset of patients with schizophrenia present clinically significant persistent negative symptoms (PNS). Identifying the neural substrates of PNS could help improve our understanding and treatment of these symptoms. Methods: This study included 64 non-affective first-episode of psychosis (FEP) patients and 60 healthy controls; 16 patients displayed PNS (i.e., at least one primary negative symptom at moderate or worse severity sustained for at least six consecutive months). Using voxel-based morphometry (VBM), we explored for gray matter differences between PNS and non-PNS patients; patient groups were also compared to controls. All comparisons were performed at $p<0.05$, corrected for multiple comparisons. Results: PNS patients had smaller gray matter in the right frontal medial-orbital gyrus (extending into the inferior frontal gyrus) and right parahippocampal gyrus (extending into the fusiform gyrus) compared to non-PNS patients. Compared to controls, PNS patients had smaller gray matter in the right parahippocampal gyrus (extending into the fusiform gyrus and superior temporal gyrus); non-PNS patients showed no significant differences to controls. Conclusion: Neural substrates of PNS are evident in FEP patients. A better understanding of the neural etiology of PNS may encourage the search for new medications and/or alternative treatments to better help those affected.
\end{abstract}

Keywords: first-episode psychosis, persistent negative symptoms, magnetic resonance imaging, voxel-based morphometry, neural substrates, frontal lobe

\section{INTRODUCTION}

In schizophrenia, negative symptoms are defined as the absence or diminution of normal behavior in the areas of affect (blunted affect, anhedonia-asociality), speech (alogia), and goal-directed behavior (avolition-apathy; Carpenter et al., 1988). Primary negative symptoms are considered intrinsic to schizophrenia while secondary negative symptoms may occur in association with or be caused by positive, depressive, or extrapyramidal symptoms (from possible side effects of neuroleptic medications; Buchanan, 2007). Patients with enduring primary negative symptoms can be classified as having deficit syndrome (DS) or persistent negative symptoms (PNS).

Briefly, DS is typically assessed using the Schedule for Deficit Syndrome (SDS) (Kirkpatrick et al., 1989). The SDS assesses: restricted affect, diminished emotional range, poverty of speech, curbing of interest, diminished sense of purpose, and diminished social drive. At least two of these symptoms must be rated "moderate" in severity or worse for 12 consecutive months to be classified with DS. The SDS rules out secondary negative symptoms by assessing anxiety, medication effects, psychotic symptoms, mental retardation, and depression. Finally, only those diagnosed with a schizophrenia spectrum disorder can be classified with DS (Kirkpatrick et al., 1989).
For a PNS classification, as in our case, negative symptoms must be present for at least six consecutive months with severity assessed in terms of need for treatment (e.g., "moderate" or worse; Buchanan, 2007). Classification can be completed using any of the accepted and validated negative symptom scales and does not require the use of a specifically developed scale (like the SDS) or access to extensive symptom history. Secondary negative symptoms are ruled out using any of the accepted and validated positive symptom scales, depression scales, and extrapyramidal symptom scales. PNS classification is not diagnosis specific and is thought to represent a broader concept than DS thus making it better suited for clinical trials (Buchanan, 2007).

With modest epidemiological data available on PNS, prevalence in schizophrenia is estimated to be above $20 \%$ (higher than that of DS; Buchanan, 2007). In fact, some first-episode of psychosis (FEP) studies reported a prevalence of severe or PNS at 23-40\% (Malla et al., 2002, 2004; Makinen et al., 2008). With increased severity of negative symptoms interfering with achievement of full remission (Andreasen et al., 2005) and negatively impacting functional outcome (Milev et al., 2005), clinically significant PNS represent an unmet therapeutic need in many cases (Kirkpatrick et al., 2006). Moreover effective drug treatments are still not available (Goff et al., 1999; Heresco-Levy et al., 1999; 
Buchanan, 2007; Buchanan et al., 2007); however, newer, more effective, treatments could be pursued based on more reliable and consistent neurobiological substrates (Kirkpatrick et al., 2006).

In schizophrenia, negative symptom severity has been related to changes in numerous brain regions including the frontal cortex (Gur et al., 2000; Lacerda et al., 2007; Nesvag et al., 2009), temporal cortex (Turetsky et al., 1995; Nesvag et al., 2009), thalamus (Preuss et al., 2005), amygdala-hippocampal complex (Rajarethinam et al., 2001; Yoshida et al., 2009), and ventricles (Andreasen et al., 1982; Saijo et al., 2001). Results, however, are inconsistent for some regions (temporal lobes, prefrontal cortex, and lateral ventricles), some studies report an association between greater gray matter loss and more severe negative symptoms but also the opposite (more gray matter loss associated with improvement in negative symptoms), while others report no significant association (Hulshoff Pol and Kahn, 2008). Moreover, no abovementioned study controlled for secondary negative symptoms, so studying DS or PNS exclusively could help reduce this confound and provide a better understanding of primary negative symptoms (Buchanan, 2007).

Following this, Kirkpatrick and Buchanan (1990) hypothesized that DS should involve a malfunction in the amygdala, peri-amygdalar cortex, and prefrontal cortex circuitry (with the same areas affected in PNS as the two sub-groupings are similar; Buchanan, 2007). Since their hypothesis, several neuroimaging studies have investigated differences between DS and non-DS patients (see Table 1 for an exhaustive summary of the main findings). Very few studies have reported brain tissue differences that reached statistical significance; in those who did, right temporal lobe (Galderisi et al., 2008) and right ventricular (Quarantelli et al., 2002) differences showed associations with primary negative symptoms (or DS). Several studies however found negative results for these same regions (right temporal lobe: Turetsky et al., 1995; Quarantelli et al., 2002; right ventricle: Gur et al., 1994; Galderisi et al., 2008). A more recent study by Cascella et al. (2010) comparing DS and non-DS patients using voxel-based morphometry (VBM) reported robust reductions in gray matter in the DS group bilaterally in the frontal and temporal lobes and in the right cingulate cortex and left putamen. Although these important studies provide guidance to the current one, it should be noted that the DS/non-DS dichotomy is conceptually and empirically different and more restrictive than the PNS/non-PNS classification used is this current investigation. Additionally, several confounds cannot be excluded from these studies, like chronicity and long-term medication effects. For example, increased volume in basal ganglia structures but not amygdala-hippocampal complex has been associated with prolonged antipsychotic exposure (Gur et al., 1998b; Lieberman et al., 2001; Glenthoj et al., 2007).

To the best of our knowledge, there are no neuroimaging studies investigating PNS. Using VBM, a fully automated technique, we set out to explore gray matter differences between non-affective FEP patients identified prospectively with PNS and those without; these two groups were also compared with healthy controls. Regarding our choice of brain structure exploration, there are several advantages to using VBM: it avoids variance between raters, which occurs when using manual segmentation of brain tissues, it is easy and quick to analyze, and it is not dependent on a particular hypothesis (Ashburner and Friston, 2001; Badcock et al., 2007). As such, VBM represents a heuristic exploratory approach which can lead to the generation of specific regions of interest. Examining PNS in a first-episode sample is more promising from a neurobiological perspective to reveal potential neural substrates of these enduring symptoms as medication and chronicity confounds are better controlled for. Additionally, since we were studying people early in the course of their illness, examining PNS over DS was preferable since it is less restrictive (Buchanan, 2007). This characteristic allowed us to identify a sub-group of patients which are at risk of developing enduring negative symptoms based on the available 1-year of clinical follow-up information. Although our analysis was exploratory, we expected smaller gray matter in the frontal and temporal regions in the PNS patients compared to the non-PNS patients based on previous findings in DS (see Table 1).

\section{MATERIALS AND METHODS PARTICIPANTS AND TREATMENT SETTING}

All patients were recruited and treated through the Prevention and Early Intervention Program for Psychoses (PEPP-Montreal), a specialized early intervention service at the Douglas Mental Health University Institute in Montreal, Canada. People aged 1530 years from the local catchment area suffering from either affective or non-affective psychosis who had not taken antipsychotic medication for more than one month with an IQ above 70 were consecutively admitted as either in- or out-patients. For complete program details see Malla et al. (2003) or visit http://www.douglasresearch.qc.ca/pages/view?section_id = 165). Those not meeting entry criteria were treated elsewhere and therefore not eligible for our neuroimaging study or any follow-up. For the neuroimaging study, only patients aged 18 to 30 years with no previous history of neurological disease or head trauma causing loss of consciousness were eligible. Furthermore, this study was limited to those with non-affective psychosis. All clinical evaluations were made by the trained clinicians working at the PEPP-Montreal clinic as part of the clinic's usual protocol, raters were not aware of the subsequent PNS categorization.

In all, 64 non-affective FEP patients were subsequently separated into two groups: PNS ( $n=16,25.0 \%)$ and non-PNS ( $n=48$, $75.0 \%)$. The criteria used for defining PNS was adapted from both Malla et al. (2004) and Buchanan (2007). PNS was defined as a global rating of moderate (3) or more on at least one negative symptom (affective flattening, alogia, avolition-apathy, or anhedonia-asociality) as measured with the Scale for the Assessment of Negative Symptoms (SANS; Andreasen, 1984a; Malla et al., 2004; Buchanan, 2007). Additionally, as suggested by Malla et al. (2004), if a score of 3 or greater was achieved on affective flattening and alogia entirely as a result of items inappropriate affect and poverty of content of speech, respectively, such patients were excluded from being classified with PNS. PNS patients had to present with primary negative symptoms, that is, PNS patients had to have a global rating of mild (2) or less on all positive symptoms as measured with the Scale for the Assessment of Positive Symptoms (SAPS; Andreasen, 1984b; Buchanan, 2007), a total score of 4 or less on the Calgary Depression Scale for Schizophrenia (CDSS; Addington et al., 1993; Malla et al., 2004; Buchanan, 2007), and low levels of extrapyramidal symptoms [i.e., symptoms were absent 
Table 1 | Summary of neuroimaging results examining deficit syndrome schizophrenia.

Left hemisphere

Right hemisphere

\begin{tabular}{|c|c|c|c|c|c|c|}
\hline \multicolumn{7}{|l|}{ FRONTAL CORTEX } \\
\hline Buchanan et al. (1993) & $\mathrm{DS}>\mathrm{NDS}$ & $\mathrm{DS}>\mathrm{C}$ & $\mathrm{NDS}<\mathrm{C}$ & $\mathrm{DS}>\mathrm{NDS}$ & $\mathrm{DS}>\mathrm{C}$ & $\mathrm{NDS}<\mathrm{C}$ \\
\hline Turetsky et al. (1995) & $\mathrm{DS}<\mathrm{NDS}$ & $\mathrm{DS}<\mathrm{C}$ & $\mathrm{NDS}>\mathrm{C}$ & $\mathrm{DS}<\mathrm{NDS}$ & $\mathrm{DS}<\mathrm{C}$ & $\mathrm{NDS}>\mathrm{C}$ \\
\hline Sigmundsson et al. $(2001)^{\mathrm{c}}$ & & $\mathrm{DS}<\mathrm{C}^{*}$ & & & $\mathrm{DS}<\mathrm{C}^{*}$ & \\
\hline Quarantelli et al. (2002) & $D S>N D S$ & $\mathrm{DS}<\mathrm{C}^{*}$ & $\mathrm{NDS}<\mathrm{C}^{*}$ & $\mathrm{DS}>\mathrm{NDS}$ & $\mathrm{DS}<\mathrm{C}^{*}$ & $\mathrm{NDS}<\mathrm{C}^{*}$ \\
\hline Galderisi et al. (2008) & $\mathrm{DS}>\mathrm{NDS}$ & $\mathrm{DS}<\mathrm{C}^{*}$ & $N D S<C^{*}$ & $\mathrm{DS}>\mathrm{NDS}$ & $\mathrm{DS}<\mathrm{C}^{*}$ & $\mathrm{NDS}<\mathrm{C}^{*}$ \\
\hline Cascella et al. (2010) & DS $<$ NDS $^{*}$ & $\mathrm{DS}<\mathrm{C}^{*}$ & $\mathrm{NDS}<\mathrm{C}^{*}$ & DS $<$ NDS $^{*}$ & $\mathrm{DS}<\mathrm{C}$ & $\mathrm{NDS}<\mathrm{C}^{*}$ \\
\hline Sigmundsson et al. (2001) & & $\mathrm{DS}<\mathrm{C}^{*}$ & & & & \\
\hline Quarantelli et al. (2002) & $\mathrm{DS}>\mathrm{NDS}$ & $\mathrm{DS}<\mathrm{C}^{*}$ & $N D S<C^{*}$ & $\mathrm{DS}>\mathrm{NDS}$ & $\mathrm{DS}<\mathrm{C}^{*}$ & $\mathrm{NDS}<\mathrm{C}^{*}$ \\
\hline Galderisi et al. (2008) & $\mathrm{DS}>\mathrm{NDS}$ & $\mathrm{DS}<\mathrm{C}^{*}$ & $\mathrm{NDS}<\mathrm{C}^{*}$ & DS $<$ NDS* & $\mathrm{DS}<\mathrm{C}^{*}$ & $\mathrm{NDS}<\mathrm{C}^{*}$ \\
\hline Cascella et al. (2010) & DS $<$ NDS $^{*}$ & $\mathrm{DS}<\mathrm{C}^{*}$ & $\mathrm{NDS}<\mathrm{C}^{*}$ & DS $<$ NDS $*$ & $\mathrm{DS}<\mathrm{C}^{*}$ & $\mathrm{NDS}<\mathrm{C}^{*}$ \\
\hline \multicolumn{7}{|l|}{ CINGULATE CORTEX } \\
\hline Galderisi et al. (2008) & $\mathrm{DS}<\mathrm{NDS}$ & $\mathrm{DS}<\mathrm{C}$ & $N D S>C$ & $\mathrm{DS}<\mathrm{NDS}$ & $\mathrm{DS}<\mathrm{C}$ & $\mathrm{NDS}>\mathrm{C}$ \\
\hline \multicolumn{7}{|l|}{ PUTAMEN } \\
\hline Sigmundsson et al. $(2001)^{\mathrm{C}}$ & & $D S>C^{*}$ & & & & \\
\hline Galderisi et al. (2008) & $\mathrm{DS}<\mathrm{NDS}$ & $\mathrm{DS}>\mathrm{C}$ & $\mathrm{NDS}>\mathrm{C}$ & $\mathrm{DS}>\mathrm{NDS}$ & $\mathrm{DS}>\mathrm{C}$ & $\mathrm{NDS}>\mathrm{C}$ \\
\hline Cascella et al. (2010)d & & & & DS $<$ NDS $^{*}$ & & \\
\hline \multicolumn{7}{|l|}{ GLOBUS PALLIDUS } \\
\hline Sigmundsson et al. $(2001)^{\mathrm{C}}$ & & $D S>C^{*}$ & & & & \\
\hline Galderisi et al. (2008) & $\mathrm{DS}<\mathrm{NDS}$ & $D S>C$ & $N D S>C$ & $\mathrm{DS}>\mathrm{NDS}$ & $\mathrm{DS}>\mathrm{C}$ & $\mathrm{NDS}>\mathrm{C}$ \\
\hline \multicolumn{7}{|c|}{ AMYGDALA-HIPPOCAMPUS } \\
\hline Buchanan et al. (1993) & $\mathrm{DS}>\mathrm{NDS}$ & $D S<C^{*}$ & $\mathrm{NDS}<\mathrm{C}^{*}$ & $\mathrm{DS}>\mathrm{NDS}$ & $\mathrm{DS}<\mathrm{C}^{*}$ & $\mathrm{NDS}<\mathrm{C}^{*}$ \\
\hline
\end{tabular}

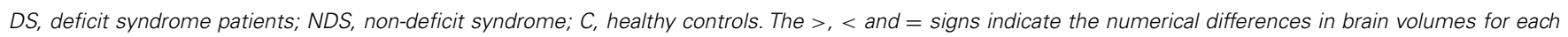

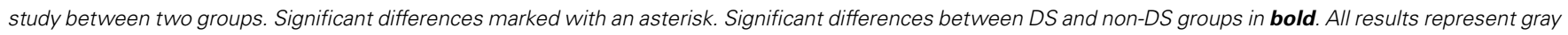
matter differences except where noted.

${ }^{a}$ Examined parenchymal volume (gray and white matter) left side: $D S>N D S *, D S<C, N D S<C * ;$ right side: $D S>N D S *, D S<C, N D S<C * ;$ for white matter left side: $D S>N D S, D S<C, N D S<C *$; right side: $D S>N D S *, D S<C, N D S<C *$

${ }^{b}$ Examined parenchymal volume only.

${ }^{c}$ Only compared DS group to healthy controls using fully automated technique so only significant results were presented.

¿Used fully automated technique so significant results were only presented.

e Did not include numerical data for this comparison.

or too mild to require treatment with anticholinergic medication based on the Extrapyramidal Symptoms Rating Scale (ESRS; Chouinard and Margolese, 2005)] (Malla et al., 2004; Buchanan, 2007). Finally, the abovementioned criteria had to be maintained for a period of at least six consecutive months (between month 6 and 12 after admission, in our case; Malla et al., 2004; Buchanan, 2007).

Among the 48 non-PNS patients, nine displayed PNS but were excluded from the PNS group because of clinically relevant positive $(n=7)$ and depressive symptoms $(n=2)$; none were excluded due to extrapyramidal symptoms. Diagnoses for patients included: schizophrenia (PNS $=12$; non$\mathrm{PNS}=34)$, schizoaffective disorder $(\mathrm{PNS}=3$; non-PNS $=7$ ), schizophreniform disorder (non-PNS $=1$ ), and psychosis NOS (PNS $=1$; non-PNS $=6$ ) according to the Structured Clinical Interview for DSM-IV (First et al., 1998) confirmed between two senior research psychiatrists (Ashok K. Malla and Ridha Joober). 
Sixty healthy controls were recruited through advertisements in local newspapers. Controls were included if they had no current or past history of (1) any Axis I disorders [as assessed with the Structured Clinical Interview for DSM-IV-TR Axis I Disorders - Nonpatient Edition (First et al., 2007)], (2) any neurological diseases, (3) head trauma causing loss of consciousness, or (4) a firstdegree family member suffering from a schizophrenia spectrum disorder. Controls were also chosen based on socio-demographic variables (age, sex, and parental-socioeconomic status) matched to all first-episode patients participating in the neuroimaging study.

Upon entry to PEPP-Montreal, written informed consent was obtained for all clinical and neurocognitive assessments done during the 2-year program to be used in a longitudinal study of early intervention in psychosis and to allow data from these assessments to be used in subsequent studies. After a comprehensive description of the present study, written informed consent was obtained from all participants. The PEPP-Montreal protocol and this research protocol were approved by the Douglas Mental Health University Institute Ethics Board and the McGill University Faculty of Medicine Review Board.

\section{SOCIO-DEMOGRAPHIC AND CLINICAL CHARACTERISTICS}

Negative and positive symptoms were assessed with the SANS (Andreasen, 1984a) and the SAPS (Andreasen, 1984b), respectively. Evaluators at PEPP have established an ICC of 0.89 on the SAPS and 0.71 on the SANS; all raters participated in inter-rater reliability sessions at least once a year to avoid rater drift. Depressive symptoms were assessed with the CDSS (Addington et al., 1993) and extrapyramidal symptoms with the ESRS (Chouinard and Margolese, 2005). If prescribed, based on the ESRS and attending physician's discretion, type and dose of anticholinergic taken were recorded. The type and dosage of antipsychotic taken were also recorded and subsequently converted into chlorpromazine equivalents (Woods, 2003; Jensen and Regier, 2010). Only one patient out of our sample was taking a typical antipsychotic and only for the first 2 months of treatment; all others were taking atypical antipsychotics for the entire treatment period. Medication adherence, based on a 5-point scale ranging from 0 (never) to 4 (fully), was obtained from patients or, when possible, from family members; following a method validated elsewhere (Cassidy et al., 2010). The abovementioned data were obtained at first assessment and at months 1, 2, 3, 6, 9, and 12 after first assessment; first assessment was conducted, on average, within 1 month after admission (in days; mean $=25.3, \mathrm{SD}=9.3$, range $=4.8-51.0$ ). Finally, the following data were acquired at first assessment: education level (number of school years completed), Full Scale IQ with the Wechsler Adult Intelligence Scale (Wechsler, 1997), parentalsocioeconomic status (SES) with the Hollingshead two-factor index (Hollingshead, 1965), and handedness with the Edinburgh Handedness Inventory (Oldfield, 1971).

Among the three groups, age at scan, education level, and Full Scale IQ were compared using a one-way ANOVA (post hoc Tukey's HSD test), parental SES with a Kruskal-Wallis $H$-test (post hoc Mann-Whitney $U$-test), and gender and handedness with cross tabulation and Chi-square tests. Between patient groups, independent $t$-tests were used to compare antipsychotic dosage, symptom totals, and duration of untreated illness and psychosis (DUI, DUP; in weeks) and Mann-Whitney $U$-tests to compare medication adherence at first assessment, month 6, and month 12. Data on CDSS and DUP were log-transformed while SAPS, antipsychotic total dosage and DUI were square-root transformed to achieve normal distribution; all other variables were normally distributed. All analyses were conducted using SPSS version 12 (SPSS, Chicago, IL, USA) and were two-tailed with a critical $p$-value of 0.05 .

\section{MRI DATA ACQUISITION AND ANALYSIS}

Scanning was carried out at the MNI on a 1.5 -T Siemens whole body MRI system. Structural T1 volumes were acquired for each participant using a three-dimensional (3D) gradient echo pulse sequence with sagittal volume excitation (repetition time $=22 \mathrm{~ms}$, echo time $=9.2 \mathrm{~ms}$, flip angle $=30^{\circ}, 1801 \mathrm{~mm}$ contiguous sagittal slices). The rectangular field-of-view for the images was $256 \mathrm{~mm}$ $(\mathrm{SI}) \times 204 \mathrm{~mm}(\mathrm{AP})$. Patient groups did not differ as to when sessions took place past entry (in weeks; $\mathrm{PNS}$ mean $=15.9, \mathrm{SD}=5.8$; non-PNS mean $=19.4, \mathrm{SD}=8.0 ; t=1.63, \mathrm{df}=62, p=0.11)$.

Structural T1 images were analyzed using VBM (Ashburner and Friston, 2000; Good et al., 2001) using software VBM8 version 369 created by C. Gaser (http://dbm.neuro.uni-jena.de/vbm/). First, the T1 images were normalized to a template space using high-dimensional (DARTEL) spatial normalization and then segmented into gray matter (GM), white matter (WM), and cerebrospinal fluid (CSF). After preprocessing, the resulting modulated images were smoothed with a $10-\mathrm{mm}$ FWHM Gaussian kernel. Gray matter (GM) volumetric differences were explored among PNS patient, non-PNS patients, and healthy controls using $t$-tests (PNS vs. non-PNS, PNS vs. controls, and non-PNS vs. controls). Our main comparison focused on the patients that included two contrasts: "non-PNS > PNS" and "PNS > non-PNS." Our secondary comparisons included contrasts against healthy controls that included the following: "control > PNS," "PNS > control," "control > non-PNS" and "non-PNS > control." All contrasts were explored using a statistical threshold of $p<0.05$, family wise error corrected for multiple comparisons. Using the MNI coordinates of the peak voxel(s) for significant clusters, structures were identified using the automated anatomical labeling toolbox (TzourioMazoyer et al., 2002). Finally, whole-brain GM, WM, and CSF volumes were estimated during the preprocessing for each participant and were summed for an estimation of total intracranial volume (TIV); the four volumes were compared among the three groups using an ANOVA (post hoc Tukey's HSD test).

\section{RESULTS}

\section{SOCIO-DEMOGRAPHIC, PSYCHOPATHOLOGY, AND MEDICATION}

See Tables 2 and 3 for values and statistical results. The three groups did not significantly differ on age, parental SES, gender, or handedness. PNS and non-PNS patients together had fewer years of education and a lower IQ compared to healthy controls; the patient groups did not significantly differ in education level or IQ.

As per design, PNS and non-PNS patients did not significantly differ on negative symptom total at first assessment whereas PNS patients had significantly higher totals at month 6 and 12. Patient groups did not significantly differ on positive symptoms totals at first assessment or month 6 but did at month 12. The higher score for the PNS patients at month 12 resulted from three patients 
Table 2 | Socio-demographic variables and brain tissue volumes.

\begin{tabular}{|c|c|c|c|c|c|c|}
\hline & PNS $(n=16)$ & non-PNS $(n=48)$ & Controls $(n=60)$ & Statistic & df & $p$ \\
\hline \multicolumn{7}{|c|}{ SOCIO-DEMOGRAPHIC VARIABLES } \\
\hline Age at scan (years) & $24.2 \pm 4.3$ & $23.6 \pm 3.4$ & $24.8 \pm 3.3$ & $F=1.61$ & 2,121 & 0.203 \\
\hline Education ${ }^{\mathrm{b}}$ & $11.2 \pm 2.0$ & $12.2 \pm 2.6$ & $14.4 \pm 2.5$ & $F=16.78$ & 2,121 & $<0.001$ \\
\hline Full scale $\mathrm{IQ}^{\mathrm{c}}$ & $97.6 \pm 18.2$ & $95.5 \pm 12.9$ & $107.9 \pm 14.9$ & $F=10.1$ & 2,119 & $<0.001$ \\
\hline \multicolumn{7}{|c|}{ BRAIN TISSUE VOLUMES (ml) } \\
\hline Gray matter & $624 \pm 56$ & $642 \pm 59$ & $658 \pm 71$ & $F=1.95$ & 2,121 & 0.147 \\
\hline White matter & $605 \pm 65$ & $596 \pm 62$ & $618 \pm 71$ & $F=1.46$ & 2,121 & 0.237 \\
\hline Cerebral-spinal fluid & $201 \pm 27$ & $198 \pm 27$ & $203 \pm 35$ & $F=0.40$ & 2,121 & 0.673 \\
\hline Total intracranial & $1430 \pm 127$ & $1436 \pm 119$ & $1479 \pm 151$ & $F=1.65$ & 2,121 & 0.197 \\
\hline
\end{tabular}

PNS, persistent negative symptoms.

${ }^{a}$ Hollingshead parental-socioeconomic status: $1=$ highest and $5=$ lowest.

${ }^{b}$ Education level measured as number of years completed; post hoc tests revealed: $P N S=$ non-PNS $(p=0.298)$; $P N S<$ controls $(p<0.001)$; non-PNS $<$ controls $(p<0.001)$.

${ }^{c}$ Full Scale 10 measured with the Wechsler Adult Intelligence Scale (data were available for 58 healthy controls); post hoc tests revealed: PNS $=$ non-PNS ( $\left.p=0.897\right)$; PNS $<$ controls $(p=0.037)$; non-PNS $<$ controls $(p<0.001)$.

Table 3 | Clinical characteristics for PNS and non-PNS patients.

\begin{tabular}{|c|c|c|c|c|}
\hline Clinical characteristics & PNS $(n=16)$ & non-PNS $(n=48)$ & Statistic & $\boldsymbol{p}$ \\
\hline \multicolumn{5}{|l|}{ NEGATIVE SYMPTOMS TOTAL (SANS) } \\
\hline First assessment & $31.2 \pm 13.6$ & $27.2 \pm 12.4$ & $t=-1.09$ & 0.280 \\
\hline 12-Month & $30.1 \pm 15.4$ & $14.8 \pm 10.0$ & $t=-4.14$ & $<0.001$ \\
\hline \multicolumn{5}{|l|}{ POSITIVE SYMPTOM TOTAL (SAPS) } \\
\hline 12-Month ${ }^{a}$ & $16.4 \pm 13.9$ & $8.6 \pm 11.7$ & $t=-2.73$ & 0.011 \\
\hline \multicolumn{5}{|l|}{ DEPRESSIVE SYMPTOM TOTAL (CDSS) } \\
\hline First assessment $^{\mathrm{a}}$ & $3.7 \pm 4.4$ & $4.8 \pm 5.2$ & $t=0.46$ & 0.645 \\
\hline 6-Montha & $2.9 \pm 3.5$ & $1.7 \pm 3.2$ & $t=-1.62$ & 0.111 \\
\hline 12-Month & $1.6 \pm 2.3$ & $1.8 \pm 3.2$ & $t=0.16$ & 0.873 \\
\hline \multicolumn{5}{|l|}{ MEDICATION ADHERENCE ${ }^{\mathrm{C}}$} \\
\hline First assessment & $3.3 \pm 1.5$ & $3.2 \pm 1.5$ & $U=362.0$ & 0.645 \\
\hline 6-Month & $3.1 \pm 1.2$ & $3.0 \pm 1.4$ & $U=379.5$ & 0.939 \\
\hline 12-Month & $2.3 \pm 1.9$ & $3.2 \pm 1.5$ & $U=294.0$ & 0.102 \\
\hline Duration of untreated illness (weeks) & $409.6 \pm 283.5$ & $281.8 \pm 250.7$ & $t=-1.76$ & 0.083 \\
\hline Duration of untreated psychosis (weeks) & $37.2 \pm 51.6$ & $62.4 \pm 84.4$ & $t=0.75$ & 0.457 \\
\hline
\end{tabular}

PNS, persistent negative symptoms.

${ }^{a}$ Analyzed using transformed data but values are presented in raw form.

${ }^{b}$ Antipsychotic total presented in chlorpromazine equivalents.

${ }^{c}$ Medication adherence: $0=$ never adherent to $4=$ fully adherent. 
relapsing on several positive symptoms driving the group average higher; removal of these people produced similar group totals (adjusted PNS mean $=11.5 \pm 9.5 ; t=-1.50, \mathrm{df}=59, p=0.14$ ). The patient groups did not significantly differ on depressive symptom totals at any time point. As well, there was no significant difference in total antipsychotic dosage (in chlorpromazine equivalents); of note, PNS patients had a much lower total at month 12 (refer to Table 4 for a more detailed account of the antipsychotic type and dosage taken by the patients over the 12-month treatment period). The two groups did not differ in medication adherence at any time point.

\section{STRUCTURAL NEUROIMAGING (VOXEL-BASED MORPHOMETRY)}

See Table 5 for all VBM results. The "non-PNS > PNS" contrast revealed significantly smaller GM in the right frontal medialorbital gyrus (extending into the inferior frontal gyrus) and right parahippocampal gyrus (extending into the fusiform gyrus) in the PNS patients relative to non-PNS patients (Figure 1); the opposite contrast ("PNS > non-PNS") revealed no significant GM differences. For the contrasts with healthy controls, the "control > PNS" contrast revealed significantly smaller GM in the right parahippocampal gyrus (extending to the superior temporal gyrus and fusiform gyrus) in PNS patients compared to controls (Figure 2). All other contrasts with controls revealed no significant differences. Finally, there were no significant between-group differences regarding whole-brain GM, WM, and CSF volumes and TIV (Table 2).

\section{SUPPLEMENTARY ANALYSES}

Although we were investigating a first-episode sample to reduce the effect antipsychotic medication exposure may have on brain morphology, patients were scanned, on average, 18 weeks past entry where all but three (two non-PNS and one PNS) had taken antipsychotic medications. Therefore, we calculated a cumulative antipsychotic dosage (in chlorpromazine equivalents) from PEPP entry until scan weighted on medication adherence (PNS mean $=458 \pm 360$; non-PNS mean $=674 \pm 561 ; t=1.44, \mathrm{df}=62$, $p=0.155)$ and re-analyzed the VBM using this as a covariate. For our main contrast of interest ("non-PNS $>$ PNS") results remained the same but with slightly different cluster sizes, $p$-values, and $t$-values. For the peak voxels in each cluster, the right frontal medial-orbital gyrus: cluster size $=2648, p$-value $<0.001$, and $t$ value $=5.03$; the right parahippocampal gyrus: cluster size $=997$, $p$-value $=0.023$, and $t$-value $=5.01$.

We also explored correlations between negative symptom totals (at first assessment, month 6, and month 12) and the GM volume estimates from the regions found in the non-PNS $>$ PNS contrast of the VBM analysis (critical $p$-value set at 0.016, 0.05/3). We found a continuous relationship between parahippocampal GM and severity of negative symptoms at month $6(r=-0.459$, $p<0.001)$ and month $12(r=-0.325, p=0.009)$; trend-level association at first assessment $(r=-0.273, p=0.029)$. Conversely, frontal GM did not show any significant linear relationship with negative symptoms at any time point.

A supplementary VBM analysis was performed using a more lenient significance threshold ( $p<0.001$, uncorrected) and we found reduced GM in PNS patients compared to controls in several frontal regions [anterior cingulate (cluster size $=36$, $t=3.31, p=0.620$ ); middle cingulate (cluster size $=44, t=3.45$, $p=0.579$ ); medial-orbital frontal gyrus (cluster size $=107$, $t=3.52, p=0.372$ ); and inferior orbital frontal gyrus (cluster size $=25, t=3.38, p=0.687)]$.

\section{DISCUSSION}

This novel study examined the neural substrates of PNS using VBM in a cohort of non-affective FEP patients. Previous studies examined changes in brain structures associated with DS in people with chronic schizophrenia using manual, semi-automated, or fully automated techniques. The prevalence of PNS in our sample was $25 \%$ - in line with the suggested prevalence of over $20 \%$ (Buchanan, 2007). Although DS and PNS differ on several aspects (Buchanan, 2007), both are fundamentally based on enduring primary negative symptoms. Our study had an advantage over the previous DS studies as our imaging data was acquired early on in the patients' treatment in an attempt to reduce the possible effects of prolonged antipsychotic exposure on brain morphology (Moncrieff and Leo, 2010); as well, we assessed the progression of negative symptoms prospectively when categorizing the PNS patients.

\section{NEURAL SUBSTRATES OF PRIMARY NEGATIVE SYMPTOMS}

Compared to non-PNS patients, PNS patients displayed significantly smaller gray matter (GM) volumes in the right medialorbital frontal gyrus extending into the inferior frontal gyrus. These results are supported by one other study (also using VBM) which found significantly lower gray matter volume in the right medial frontal gyrus in DS compared to non-DS patients (Cascella et al., 2010); this study also found significantly smaller clusters of GM in the superior frontal gyrus bilaterally and the left middle frontal gyrus. Previous studies investigating frontal cortex volume as a whole did not report any significant differences between DS and non-DS patients (Buchanan et al., 1993; Turetsky et al., 1995; Quarantelli et al., 2002; Galderisi et al., 2008). Moreover, one of these studies reported smaller parenchymal (gray matter + white matter) volume bilaterally in the in DS patients compared to nonDS patients (Turetsky et al., 1995) while the other three reported larger frontal lobe volume bilaterally in DS patients compared to non-DS patients (Buchanan et al., 1993; Quarantelli et al., 2002; Galderisi et al., 2008) - contrasting with the directionality of our finding. Nonetheless, this is supported by the findings of Cascella et al. (2010), which also used a VBM analysis and identified significant differences in more localized areas of the frontal cortex. So, perhaps the neural substrates of primary negative symptoms in relation to the frontal cortex are more localized rather than encompassing the entire frontal area.

The PNS patients also displayed smaller GM in the right parahippocampal gyrus extending into the fusiform gyrus compared to non-PNS patients. This supported Cascella et al. (2010), albeit in different clusters, who reported smaller GM in the right inferior and middle temporal gyri and in the superior temporal gyrus bilaterally in DS patients compared to non-DS patients. Although our study did not report the same peak clusters, we did find differences specific to Brodmann Area 20. Furthermore, further examination of the figural result from Cascella et al. (2010) 
Table 4 | Types of antipsychotic medication for PNS and non-PNS patients.

First assessment

\begin{tabular}{l} 
\\
\hline Risperidone \\
Olanzapine \\
Seroquel \\
Ziprasidone \\
Paliperidone \\
Luxapac
\end{tabular}

\section{MONTH 1}

Risperidone

Olanzapine

Seroquel

Ziprasidone

Paliperidone

Haloperidol

Consta $(2 \times /$ month)

None

\section{MONTH 2}

Risperidone

Olanzapine

Seroquel

Ziprasidone

Paliperidone

Haloperidol

Consta ( $2 \times /$ month)

None

\section{MONTH 3}

Risperidone

Olanzapine

Seroquel

Ziprasidone

Paliperidone

Haloperidol

Consta $(2 \times /$ month)

None

\section{MONTH 6}

Risperidone

Olanzapine

Seroquel

Ziprasidone

Paliperidone

Haloperidol

Consta ( $2 \times /$ month)

None

\section{MONTH 9}

Risperidone

Olanzapine

Seroquel

Ziprasidone

Paliperidone
PNS

1st
$2.2(1.6)[8]$
$13.5(3.8)[5]$
$150.0[1]$
-
-
-
$[2]$

[2]

$2.3(0.6)[8]$
$12.5(3.5)[5]$
$300.0[1]$
-
-
-
$25.0[1]$
$[1]$

[1]

$2.7(1.6)[8]$
$10.5(5.4)[5]$
$300.0[1]$
-
-
-
$25.0[1]$
$[1]$

$2.2(0.8)[8]$
$11.5(7.6)[5]$
$450.0[1]$
-
-
-
$37.5[1]$
$[1]$

$\begin{array}{ll}2.4(1.4)[7] & 2.5[1] \\ 3.8(1.8)[2] & 25.0[1] \\ 250.0(70.7)[2] & - \\ - & - \\ 6.0[1] & - \\ - & - \\ 29.2(7.2)[3] & - \\ {[1]} & {[14]}\end{array}$

$\begin{array}{lll}2.1(0.5)[6] & 1.0[1] & 1.7(0.7)[16] \\ 3.8(1.8)[2] & - & 8.9(4.0)[12] \\ 325.0(35.4)[2] & - & 414.3(217.4)[7] \\ - & - & 120.0[1] \\ 3.0[1] & - & 3.0[1]\end{array}$

non-PNS

non-PNS

1st 2n

1.9 (1.1) [17]

14.9 (6.8) [18]

325.0 (176.8) [2]

80.0 [1]

$3.0(0.0)$ [2]

[8]

\section{2nd}

$2.2(1.2)[16]$
$13.8(8.3)[19]$
$300.0(230.9)[4]$
$80.0(0.0)[2]$
$3.0(0.0)[2]$
$2.0[1]$
$25.0[1]$
$[3]$

[3]

$2.1(1.1)[15]$
$11.5(7.0)[20]$
$412.5(193.1)[4]$
$140.0(28.3)[2]$
$3.0[1]$
$1.5[1]$
$31.3(8.8)[2]$
$[3]$

$\begin{array}{ll}- & 1.9(0.89)[15] \\ 2.5[1] & 11.6(5.8)[19] \\ - & 320.0(164.3)[5] \\ - & 110.0(70.7)[2] \\ - & 3.0[1] \\ - & 1.5[1] \\ - & 31.3(8.8)[2] \\ {[15]} & {[3]}\end{array}$

\begin{tabular}{|c|c|c|}
\hline \multirow[b]{2}{*}{ 2nd } & \multicolumn{2}{|c|}{$\begin{array}{l}\text { PNS vs. non-PNS } \\
\text { (1st AP only) }\end{array}$} \\
\hline & $T$ & $p$ \\
\hline- & -0.58 & 0.568 \\
\hline - & 0.42 & 0.677 \\
\hline- & & \\
\hline- & & \\
\hline- & & \\
\hline $10.0[1]$ & & \\
\hline [47] & & \\
\hline
\end{tabular}

[47]

5.0
20.0
200
-
-
-
-
$[45]$

[45]

$\begin{array}{lll}4.0[1] & -1.10 & 0.283 \\ - & 0.30 & 0.770 \\ - & & \\ - & & \\ - & & \\ - & & \\ 25.0[1] & & \\ {[46]} & \end{array}$

$\begin{array}{lll}5.0[1] & -0.11 & 0.917 \\ 20.0[1] & 0.34 & 0.737 \\ 200.0[1] & & \end{array}$


Table 4 | Continued

\begin{tabular}{|c|c|c|c|c|c|c|}
\hline \multirow[t]{2}{*}{ First assessment } & \multicolumn{2}{|c|}{ PNS } & \multicolumn{2}{|c|}{ non-PNS } & \multicolumn{2}{|c|}{$\begin{array}{l}\text { PNS vs. non-PNS } \\
\text { (1st AP only) }\end{array}$} \\
\hline & 1st & 2nd & $1 s t$ & 2nd & $T$ & $p$ \\
\hline Haloperidol & - & - & $1.5[1]$ & - & & \\
\hline Consta $(2 \times /$ month $)$ & $28.1(6.3)[4]$ & - & $30.0(68)[5]$ & - & 0.42 & 0.685 \\
\hline None & {$[1]$} & {$[15]$} & {$[5]$} & {$[44]$} & & \\
\hline \multicolumn{7}{|l|}{ MONTH 12} \\
\hline Risperidone & $2.0(0.6)[7]$ & - & $1.8(1.2)[15]$ & $2.1(0.6)[4]$ & -0.39 & 0.701 \\
\hline Olanzapine & $3.8(1.8)[2]$ & - & $7.8(4.3)[12]$ & $5.0[1]$ & 1.29 & 0.221 \\
\hline Seroquel & - & - & $483.3(194.1)[6]$ & 316.7 (275.4) [3] & & \\
\hline Ziprasidone & - & - & $100.0[1]$ & - & & \\
\hline Paliperidone & - & - & $3.0(0.0)[2]$ & - & & \\
\hline Haloperidol & - & - & $15[1]$ & - & & \\
\hline Consta ( $2 \times /$ month) & $29.2(7.2)[3]$ & $37.5[1]$ & $28.6(6.1)[7]$ & - & -0.14 & 0.896 \\
\hline Consta $(3 \times /$ month) & $25.0[1]$ & - & - & - & & \\
\hline None & [1] & [15] & {$[6]$} & {$[40]$} & & \\
\hline
\end{tabular}

Table 5 | Gray matter differences among PNS patients, non-PNS patients, and healthy controls using voxel-based morphometry.

\begin{tabular}{|c|c|c|c|c|c|c|c|}
\hline \multirow[t]{2}{*}{ Cluster size } & \multirow[t]{2}{*}{$p$-value (FWE) } & \multirow[t]{2}{*}{$t$-value } & \multicolumn{3}{|c|}{ MNI coordinates } & \multirow[t]{2}{*}{ Side } & \multirow[t]{2}{*}{ Region or structure (BA) } \\
\hline & & & $x$ & $y$ & $z$ & & \\
\hline \multirow[t]{3}{*}{1802} & 0.004 & 4.96 & 3 & 54 & -12 & Right & Medial-orbital frontal gyrus (BA 11 \\
\hline & & 4.39 & 17 & 51 & -5 & Right & Medial-orbital frontal gyrus (BA 10 \\
\hline & & 3.80 & 14 & 41 & -15 & Right & Inferior frontal gyrus (BA 11) \\
\hline 926 & & 4.21 & 39 & -33 & -17 & Right & Fusiform gyrus (BA 20) \\
\hline \multicolumn{8}{|c|}{ PNS $>$ NON-PNS } \\
\hline \multicolumn{8}{|c|}{ No significant differences } \\
\hline \multicolumn{8}{|c|}{ CONTROL > PNS } \\
\hline \multirow[t]{2}{*}{2322} & 0.002 & 4.93 & 35 & -16 & -30 & Right & Parahippocampal gyrus (BA 36) \\
\hline & & 4.64 & 39 & -31 & -17 & Right & Fusiform gyrus (BA 20) \\
\hline
\end{tabular}

PNS > CONTROL

No significant differences

CONTROL > NON-PNS

No significant differences

NON-PNS > CONTROL

No significant differences

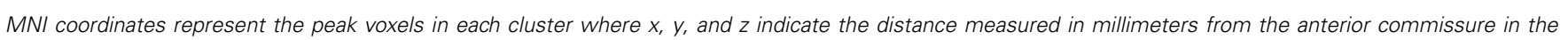
sagittal, coronal, and axial planes, respectively. BA represents the Brodmann Area for each region or structure.

revealed differences in the temporal area extended into the medialtemporal lobe (including the parahippocampal cortex bilaterally), although no significant peak was identified within these regions. Of the previous studies investigating temporal lobe volume as a whole, only one study identified a significantly smaller volume on the right side in DS patients compared to non-DS patients but not in the left side (Galderisi et al., 2008); the other two studies reported numerically smaller (Turetsky et al., 1995) and larger
(Quarantelli et al., 2002) volumes in the DS patients compared to non-DS patients. So, the neural correlates of primary negative symptoms in relation to the temporal lobe appear to be more lateralized toward the right side that may be related to the lobe as a whole or to more specific clusters therein; more studies are needed to lend support and expand upon this.

Finally, when compared to a matched sample of healthy subjects, the non-PNS patients showed no significant GM differences. 


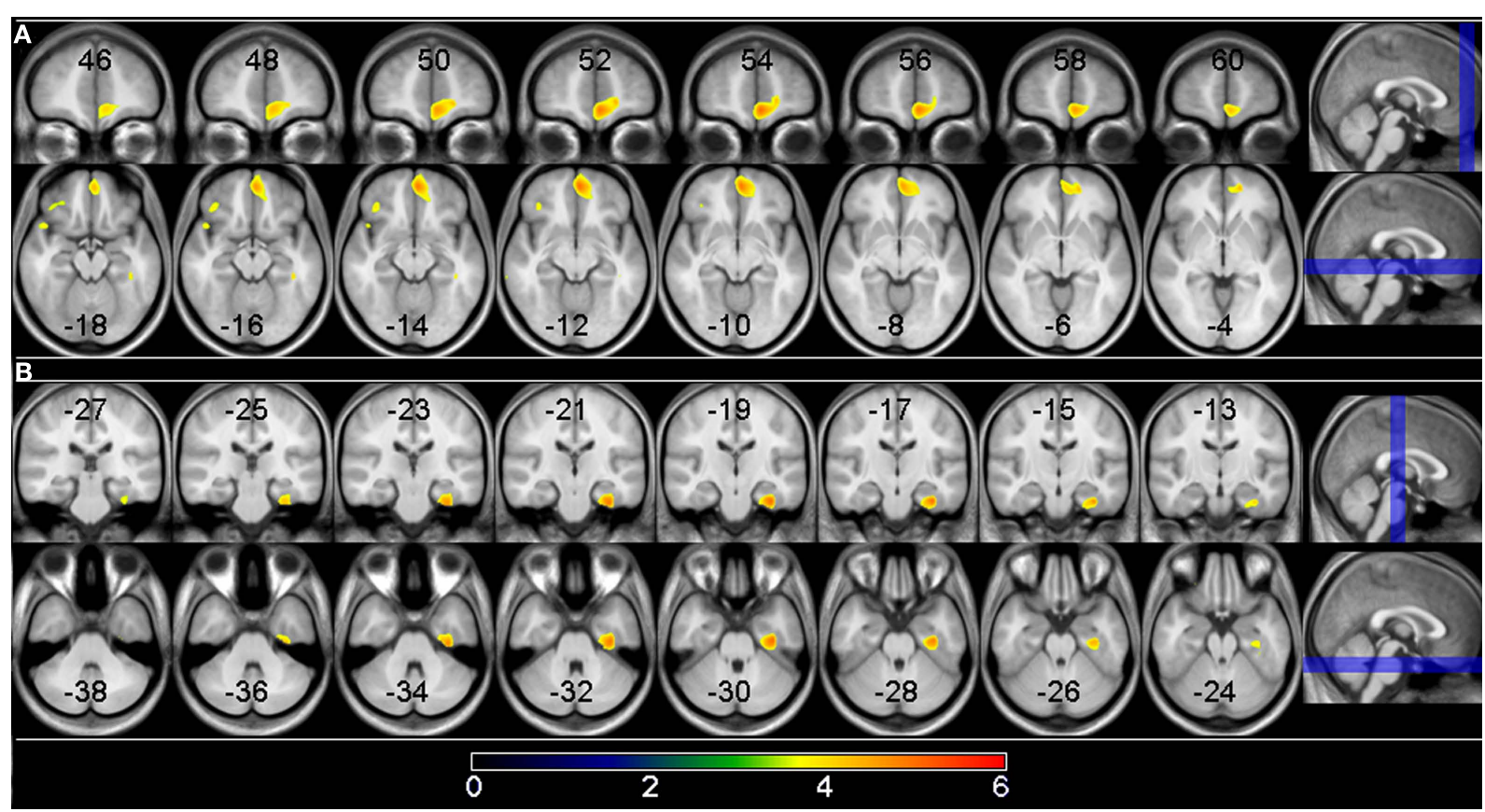

FIGURE 1 | Gray matter differences between PNS and non-PNS patients. Voxels where significant group differences were observed are depicted in color and are superimposed on a T1 MRI template image. The highlighted areas indicate smaller gray matter volume in PNS patients compared to non-PNS patients at a significance level of $p<0.05$, cluster corrected. (A) Shows the right medial frontal gyrus result; (B) shows the right parahippocampal/fusiform gyrus result. The location of the eight coronal and axial slices are illustrated on the sagittal planes with coordinates presented in MNI space. The color bar indicates the $t$-value.

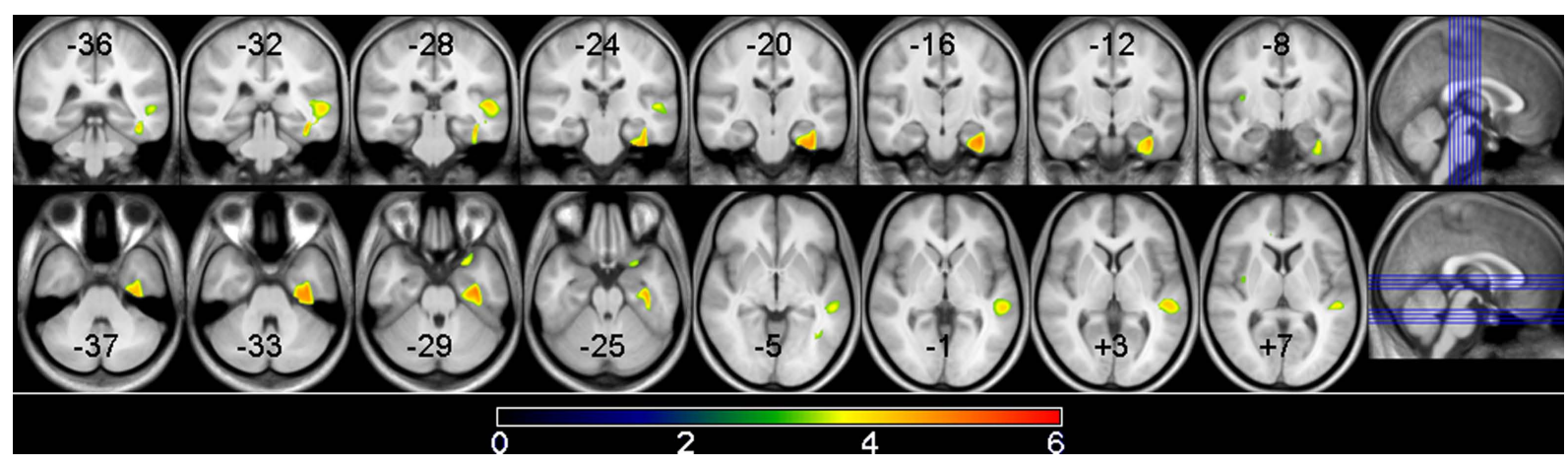

FIGURE 2 | Gray matter volume differences between PNS patients and controls. Voxels where significant group differences were observed are depicted in color and are superimposed on a T1 MRI template image. The highlighted areas indicate smaller gray matter volume in PNS patients compared to non-PNS patients at a significance level of $p<0.05$, cluster corrected. Shown here is the right parahippocampal gyrus/superior temporal gyrus result. The location of the 8 coronal and axial slices are illustrated on the sagittal planes with coordinates presented in MNI space. The color bar indicates the $t$-value.
On the other hand, the PNS patients, compared to the healthy subjects, showed smaller GM in the right parahippocampal gyrus extending into the fusiform gyrus and superior temporal gyrus (almost this exact cluster was identified when compared to the non-PNS patients). One other study found significantly smaller GM in the parahippocampal gyrus, albeit on the left side, in DS patients compared to controls (Sigmundsson et al., 2001). Three other studies found significantly smaller GM in the temporal lobe bilaterally in DS patients compared to controls (Quarantelli et al., 2002; Galderisi et al., 2008; Cascella et al., 2010). In the DS patients, one other study reported significantly smaller GM in the middle temporal gyrus bilaterally (Cascella et al., 2010) while the other two reported smaller volume in the temporal lobe as a whole (Quarantelli et al., 2002; Galderisi et al., 2008). Together, these findings suggest that smaller GM in the temporal lobe appear to be a substrate of non-affective psychosis (namely schizophrenia) with a strong relationship to primary negative symptoms. 
Overall, our results showed localized GM differences in the right medial orbitofrontal and right medial-temporal areas related to PNS which are observable early on in the course of treatment. Moreover, there were no global GM differences among patients with PNS, patients without PNS and control subjects. Therefore, specific frontotemporal variations appear to be associated with persistent primary negative symptoms in non-affective FEP patients partially supporting the original hypothesis put forward by Kirkpatrick and Buchanan (1990) that associated DS with variations in the amygdala, peri-amygdalar, and prefrontal cortex circuitry (Kirkpatrick and Buchanan, 1990). While our results implicated the prefrontal cortex with PNS, we did not find support for an association with the peri-amygdaloid or amgydaloid area. However, GM differences were identified in the parahippocampal gyrus - a region that is known to have strong connections with the peri-amygdaloid and amygdaloid area (Majak and Pitkanen, 2003). Moreover, the orbital frontal cortex along with sub-cortical regions including the amygdala-hippocampal area has been suggested to mediate negative symptoms (Zald and Kim, 2001).

Regarding the frontal area, previous studies have reported smaller GM volumes to be correlated with greater severity of negative symptoms. More specifically, these studies found associations with GM volume in the right inferior frontal opercular and triangularis (Berge et al., 2011), left straight gyrus (Szendi et al., 2006), and the orbitofrontal (Baare et al., 1999) - the region we identified to be smaller in the PNS patients compared to the nonPNS patients. On the other hand, regarding the medial-temporal lobe, several studies have related GM volume to negative symptoms as well (Gur et al., 1998a; Anderson et al., 2002; Bodnar et al., 2011). These results together suggest a strong relationship between frontal and medial-temporal GM and negative symptoms but the relationship appears stronger with the frontal region. To examine this further we performed correlations between negative symptom totals (at first assessment, month 6, and month 12) and the GM volume estimates from the medial-orbital frontal and parahippocampal regions. Only parahippocampal GM was significantly correlated with the severity of negative symptoms at month 6 and month 12 (with a trend-level result at first assessment), which may be indicative of a distinct neurobiological difference between PNS and non-PNS patients.

\section{TREATING PNS}

People with PNS have benefited from treatments like cognitive behavioral therapy (Rector et al., 2003; Turkington et al., 2008) and loving-kindness mediation (Johnson et al., 2009) in reducing negative symptoms. However, for those resistant to such treatments, alternatives must be considered like cognitive remediation or transcranial magnetic stimulation (TMS), for example. Both the frontal and temporal cortex could be targeted in pharmaco-fMRI research (Stein, 2001; Honey and Bullmore, 2004) to help identify new medications to better treat negative symptoms than the currently used agents like d-cycloserine or glycine that have produced somewhat inconsistent results (Goff et al., 1999; Heresco-Levy et al., 1999; Buchanan et al., 2007). This study suggests that the frontotemporal circuitry might be related to PNS and should be explored further as regions of interest in the development or implementation of future treatment options such as those.

\section{LIMITATIONS}

There are several limitations in our study. First, although we employed a first-episode sample in an attempt to reduce the effect of antipsychotic exposure on brain morphology (Moncrieff and Leo, 2010), the majority of patients were still treated with antipsychotics possibly affecting our results. Our main results did not change after re-analyzing the data including a cumulative antipsychotic covariate; however, examining antipsychotic naïve patients would be best to remove any effects. Second, avolition and anhedonia were more prevalent than alogia and affective flattening in our sample and, as such, our results may not be generalizable to all negative symptoms. Furthermore, the categorical approach of this study did not allow us to specify which negative symptoms contributed the most to the structural differences identified; future studies need to examine these symptoms separately. Third, we did not find any structural differences in the frontal area which has been a hallmark in studies examining schizophrenia (Shenton et al., 2001; Williams and Castner, 2008). This may have been due to the conservative statistical threshold ( $p<0.05$, family wise error corrected for multiple comparisons) we used in order to limit the chances of false positives in our VBM results. Nevertheless, when lowering our threshold ( $p<0.001$, uncorrected), we found reduced GM in PNS patients compared to controls in the anterior cingulate, middle cingulate, medial-orbital frontal gyrus, and inferior orbital frontal gyrus. These are interesting findings but the identified clusters were very small and $p$-values indicated a high probability of false positives. Fourth, the interrater score measured for the SANS is quite low (0.71) although similar to what was reported in a previous study of comparative inter-rater reliability (Norman et al., 1996). Fifth, at the time of analysis, clinical data was only available for the first 12 months of treatment in our sample; however it has been previously shown that PNS categorization is more consistent after the first year of treatment (Chang et al., 2011). In our sample we have used data from the last 6 months of treatment to limit the possible effects of the instability of symptoms but it would be interesting to re-evaluate the categorization with longer followup period. Sixth, the PNS classification being relatively new is evaluated following a set of guidelines rather than a consensus definition, which has yet to be developed. For example, the guidelines recommend PNS categorization to be given if negative symptoms are of moderate severity or worse (Buchanan, 2007), which can be based on a global rating or on ratings for specific symptoms. Lastly, our PNS patient group was relatively small, limiting the generalization (interpretation) of our results. Additionally, we could not examine whether the structural differences related to PNS were specific to one diagnosis or not because we were limited by the small number of patients with PNS that did not allow for any meaningful diagnosis specific between-group comparisons.

\section{CONCLUSION}

The current study identified smaller gray matter in the right frontal and temporal cortices in non-affective FEP patients with PNS 
compared to patients without PNS. Compared to controls, we also identified smaller GM in the temporal cortex in PNS patients with no significant GM differences with the non-PNS patients. This suggests that patients, who in the course of their illness show PNS, differ neurologically from those who do not show PNS in specific neuroanatomical regions. This subtyping of whether biological heterogeneity underlies observed clinical heterogeneity within the patient group is merely an extension of the notion that biological differences underlie observed behavioral differences between patients and controls (Turetsky et al., 1995). Nevertheless, those

\section{REFERENCES}

Addington, D., Addington, J., and Maticka-Tyndale, E. (1993). Assessing depression in schizophrenia: the Calgary Depression Scale. Br. J. Psychiatry Suppl. 22, 39-44.

Anderson, J. E., Wible, C. G., McCarley, R. W., Jakab, M., Kasai, K., and Shenton, M. E. (2002). An MRI study of temporal lobe abnormalities and negative symptoms in chronic schizophrenia. Schizophr. Res. 58, 123-134.

Andreasen, N. C. (1984a). Modified Scale for the Assessment of Negative Symptoms (SANS). Iowa City: University of Iowa.

Andreasen, N. C. (1984b). Scale for the Assessment of Positive Symptoms (SAPS). Iowa City: University of Iowa.

Andreasen, N. C., Carpenter, W. T. Jr., Kane, J. M., Lasser, R. A., Marder, S. R., and Weinberger, D. R. (2005). Remission in schizophrenia: proposed criteria and rationale for consensus. Am. J. Psychiatry 162, 441-449.

Andreasen, N. C., Olsen, S. A., Dennert, J. W., and Smith, M. R. (1982). Ventricular enlargement in schizophrenia: relationship to positive and negative symptoms. Am. J. Psychiatry 139, 297-302.

Ashburner, J., and Friston, K. J. (2000). Voxel-based morphometry - the methods. Neuroimage 11, 805-821.

Ashburner, J., and Friston, K. J. (2001). Why voxel-based morphometry should be used. Neuroimage 14, 1238-1243.

Baare, W. F., Hulshoff Pol, H. E., Hijman, R., Mali, W. P., Viergever, M. A., and Kahn, R. S. (1999). Volumetric analysis of frontal lobe regions in schizophrenia: relation to cognitive function and symptomatology. Biol. Psychiatry 45, 1597-1605.

Badcock, J. C., Fukuda, M., Gottesmann, C., Iwanami, A., Kasai, K., Lerner, V., Maybery, M. T., Miodownik, C., Moore, K., Nakagome, K., Pinikahana, J., Rogers, M. A., Sollberger, D., Walkup, J., Waters, F. A. V., and Yamasue, H. (2007). Psychology and
Schizophrenia. New York: Nova Science Publishers, Inc.

Berge, D., Carmona, S., Rovira, M., Bulbena, A., Salgado, P., and Vilarroya, O. (2011). Gray matter volume deficits and correlation with insight and negative symptoms in firstpsychotic-episode subjects. Acta Psychiatr. Scand. 123, 431-439.

Bodnar, M., Harvey, P. O., Malla, A. K., Joober, R., and Lepage, M. (2011). The parahippocampal gyrus as a neural marker of early remission in first-episode psychosis: a voxel-based morphometry study. Clin. Schizophr. Relat. Psychoses 4, 217-228.

Buchanan, R. W. (2007). Persistent negative symptoms in schizophrenia: an overview. Schizophr. Bull. 33, 1013-1022.

Buchanan, R. W., Breier, A., Kirkpatrick, B., Elkashef, A., Munson, R. C., Gellad, F., and Carpenter, W. T. Jr. (1993). Structural abnormalities in deficit and nondeficit schizophrenia. Am. J. Psychiatry 150, 59-65.

Buchanan, R. W., Javitt, D. C., Marder, S. R., Schooler, N. R., Gold, J. M., McMahon, R. P., Heresco-Levy, U., and Carpenter, W. T. (2007). The cognitive and negative symptoms in schizophrenia trial (CONSIST): the efficacy of glutamatergic agents for negative symptoms and cognitive impairments. Am. J. Psychiatry 164, 1593-1602.

Carpenter, W. T. Jr., Heinrichs, D. W., and Wagman, A. M. (1988). Deficit and nondeficit forms of schizophrenia: the concept. Am. J. Psychiatry 145, 578-583.

Cascella, N. G., Fieldstone, S. C., Rao, V. A., Pearlson, G. D., Sawa, A., and Schretlen, D. J. (2010). Gray-matter abnormalities in deficit schizophrenia. Schizophr. Res. 120, 63-70.

Cassidy, C. M., Rabinovitch, M., Schmitz, N., Joober, R., and Malla, A. (2010). A comparison study of multiple measures of adherence to antipsychotic medication in firstepisode psychosis. J. Clin. Psychopharmacol. 30, 64-67.

identified with PNS should be studied more closely as they can offer better insights in the pathophysiology of primary negative symptoms in schizophrenia and could aid in the development of more effective treatments.

\section{ACKNOWLEDGMENTS}

We thank the PEPP-Montreal research staff for their help with recruitment and clinical assessments of patients. We also wish to thank CIHR and the Sackler Foundation for their financial support of this research project.

Chang, W. C., Hui, C. L., Tang, J. Y., Wong, G. H., Lam, M. M., Chan, S. K., and Chen, E. Y. (2011). Persistent negative symptoms in first-episode schizophrenia: a prospective threeyear follow-up study. Schizophr. Res. 133, 22-28.

Chouinard, G., and Margolese, H. C. (2005). Manual for the extrapyramidal symptom rating scale (ESRS). Schizophr. Res. 76, 247-265.

First, M. B., Spitzer, R. L., Gibbon, M. and Williams, J. B. W. (1998). Structured Clinical Interview for DSMIV Axis I Disorders, Patient Edition (SCID-I/P \& SCID-I/NP), Version 2. New York: New York Psychiatric Institute, Biometrics Research.

First, M. B., Spitzer, R. L., Gibbon, M., and Williams, J. B. W. (2007). Structured Clinical Interview for DSM-IVTR Axis I Disorders - Non-patient Edition (SCID-I/NP, 1/2007 revision). New York, NY: New York State Psychiatric Institute.

Galderisi, S., Quarantelli, M., Volpe, U., Mucci, A., Cassano, G. B., Invernizzi, G., Rossi, A., Vita, A., Pini, S., Cassano, P., Daneluzzo, E., De Peri, L., Stratta, P., Brunetti, A., and Maj, M. (2008). Patterns of structural MRI abnormalities in deficit and nondeficit schizophrenia. Schizophr. Bull. 34, 393-401.

Glenthoj, A., Glenthoj, B. Y., Mackeprang, T., Pagsberg, A. K., Hemmingsen, R. P., Jernigan, T. L., and Baare, W. F. (2007). Basal ganglia volumes in drug-naive first-episode schizophrenia patients before and after short-term treatment with either a typical or an atypical antipsychotic drug. Psychiatry Res. 154, 199-208.

Goff, D. C., Tsai, G., Levitt, J., Amico, E., Manoach, D., Schoenfeld, D. A., Hayden, D. L., McCarley, R., and Coyle, J. T. (1999). A placebo-controlled trial of D-cycloserine added to conventional neuroleptics in patients with schizophrenia. Arch. Gen. Psychiatry 56, 21-27.

Good, C. D., Johnsrude, I. S., Ashburner, J., Henson, R. N., Friston, K. J., and
Frackowiak, R. S. (2001). A voxelbased morphometric study of ageing in 465 normal adult human brains. Neuroimage 14, 21-36.

Gur, R. E., Cowell, P., Turetsky, B. I., Gallacher, F., Cannon, T., Bilker, W., and Gur, R. C. (1998a). A follow-up magnetic resonance imaging study of schizophrenia. Relationship of neuroanatomical changes to clinical and neurobehavioral measures. Arch. Gen. Psychiatry 55, 145-152.

Gur, R. E., Maany, V., Mozley, P. D., Swanson, C., Bilker, W., and Gur, R. C. (1998b). Subcortical MRI volumes in neuroleptic-naive and treated patients with schizophrenia. Am. J. Psychiatry 155, 1711-1717.

Gur, R. E., Cowell, P. E., Latshaw, A., Turetsky, B. I., Grossman, R. I., Arnold, S. E., Bilker, W. B., and Gur, R. C. (2000). Reduced dorsal and orbital prefrontal gray matter volumes in schizophrenia. Arch. Gen. Psychiatry 57, 761-768.

Gur, R. E., Mozley, P. D., Shtasel, D. L., Cannon, T. D., Gallacher, F., Turetsky, B., Grossman, R., and Gur, R. C. (1994). Clinical subtypes of schizophrenia: differences in brain and CSF volume. Am. J. Psychiatry 151, 343-350.

Heresco-Levy, U., Javitt, D. C., Ermilov, M., Mordel, C., Silipo, G., and Lichtenstein, M. (1999). Efficacy of high-dose glycine in the treatment of enduring negative symptoms of schizophrenia. Arch. Gen. Psychiatry 56, 29-36.

Hollingshead, A. (1965). Two-Factor Index of Social Position. New Haven, $\mathrm{CN}$ : Yale University Press.

Honey, G., and Bullmore, E. (2004). Human pharmacological MRI Trends Pharmacol. Sci. 25, 366-374.

Hulshoff Pol, H. E., and Kahn, R. S. (2008). What happens after the first episode? A review of progressive brain changes in chronically ill patients with schizophrenia. Schizophr. Bull. 34, 354-366.

Jensen, B., and Regier, L. D. (ed.). (2010). Drug Comparison Charts, 7 th Edn. Saskatoon, SK: RxFiles Academic Detailing Program. 
Johnson, D. P., Penn, D. L., Fredrickson, B. L., Meyer, P. S., Kring, A. M., and Brantley, M. (2009). Lovingkindness meditation to enhance recovery from negative symptoms of schizophrenia. J. Clin. Psychol. 65, 499-509.

Kirkpatrick, B., and Buchanan, R. W. (1990). The neural basis of the deficit syndrome of schizophrenia. J. Nerv. Ment. Dis. 178, 545-555.

Kirkpatrick, B., Buchanan, R. W., McKenney, P. D., Alphs, L. D., and Carpenter, W. T. Jr. (1989). The schedule for the deficit syndrome: an instrument for research in schizophrenia. Psychiatry Res. 30, 119-123.

Kirkpatrick, B., Fenton, W. S., Carpenter, W. T. Jr., and Marder, S. R. (2006). The NIMH-MATRICS consensus statement on negative symptoms. Schizophr. Bull. 32, 214-219.

Lacerda, A. L., Hardan, A. Y., Yorbik, O., Vemulapalli, M., Prasad, K. M., and Keshavan, M. S. (2007). Morphology of the orbitofrontal cortex in first-episode schizophrenia: relationship with negative symptomatology. Prog. Neuropsychopharmacol. Biol. Psychiatry 31, 510-516.

Lieberman, J., Chakos, M., Wu, H., Alvir, J., Hoffman, E., Robinson, D., and Bilder, R. (2001). Longitudinal study of brain morphology in first episode schizophrenia. Biol. Psychiatry 49, 487-499.

Majak, K., and Pitkanen, A. (2003). Projections from the periamygdaloid cortex to the amygdaloid complex, the hippocampal formation, and the parahippocampal region: a PHA-L study in the rat. Hippocampus 13, 922-942.

Makinen, J., Miettunen, J., Isohanni, M., and Koponen, H. (2008). Negative symptoms in schizophrenia: a review. Nord. J. Psychiatry 62, 334-341.

Malla, A., Norman, R., McLean, T., Scholten, D., and Townsend, L. (2003). A Canadian programme for early intervention in non-affective psychotic disorders. Aust. N. Z. J. Psychiatry 37, 407-413.

Malla, A. K., Norman, R. M., Takhar, J., Manchanda, R., Townsend, L., Scholten, D., and Haricharan, R. (2004). Can patients at risk for persistent negative symptoms be identified during their first episode of psychosis? J. Nerv. Ment. Dis. 192, 455-463.

Malla, A. K., Takhar, J. J., Norman, R. M., Manchanda, R., Cortese, L., Haricharan, R., Verdi, M., and Ahmed, R. (2002). Negative symptoms in first episode non-affective psychosis. Acta Psychiatr. Scand. 105, 431-439.
Milev, P., Ho, B. C., Arndt, S., and Andreasen, N. C. (2005). Predictive values of neurocognition and negative symptoms on functional outcome in schizophrenia: a longitudinal first-episode study with 7-year follow-up. Am. J. Psychiatry 162, 495-506.

Moncrieff, J., and Leo, J. (2010). A systematic review of the effects of antipsychotic drugs on brain volume. Psychol. Med. 40, 1409-1422.

Nesvag, R., Saetre, P., Lawyer, G., Jonsson, E. G., and Agartz, I. (2009). The relationship between symptom severity and regional cortical and grey matter volumes in schizophrenia. Prog. Neuropsychopharmacol. Biol. Psychiatry 33, 482-490.

Norman, R. M., Malla, A. K., Cortese, L., and Diaz, F. (1996). A study of the interrelationship between and comparative interrater reliability of the SAPS, SANS and PANSS. Schizophr. Res. 19, 73-85.

Oldfield, R. C. (1971). The assessment and analysis of handedness: the Edinburgh inventory. Neuropsychologia 9, 97-113.

Preuss, U. W., Zetzsche, T., Jager, M., Groll, C., Frodl, T., Bottlender, R., Leinsinger, G., Hegerl, U., Hahn, K., Moller, H. J., and Meisenzahl, E. M. (2005). Thalamic volume in firstepisode and chronic schizophrenic subjects: a volumetric MRI study. Schizophr. Res. 73, 91-101.

Quarantelli, M., Larobina, M., Volpe, U., Amati, G., Tedeschi, E., Ciarmiello, A., Brunetti, A., Galderisi, S., and Alfano, B. (2002). Stereotaxybased regional brain volumetry applied to segmented MRI: validation and results in deficit and nondeficit schizophrenia. Neuroimage 17, 373-384.

Rajarethinam, R., DeQuardo, J. R., Miedler, J., Arndt, S., Kirbat, R., Brunberg, J. A., and Tandon, R. (2001). Hippocampus and amygdala in schizophrenia: assessment of the relationship of neuroanatomy to psychopathology. Psychiatry Res. 108, 79-87.

Rector, N. A., Seeman, M. V., and Segal, Z. V. (2003). Cognitive therapy for schizophrenia: a preliminary randomized controlled trial. Schizophr. Res. 63, 1-11.

Saijo, T., Abe, T., Someya, Y., Sassa, T., Sudo, Y., Suhara, T., Shuno, T., Asai, K., and Okubo, Y. (2001). Ten year progressive ventricular enlargement in schizophrenia: an MRI morphometrical study. Psychiatry Clin. Neurosci. 55, 41-47.

Shenton, M. E., Dickey, C. C., Frumin, M., and McCarley, R. W. (2001). A review of MRI findings in schizophrenia. Schizophr. Res. 49, 1-52.

Sigmundsson, T., Suckling, J., Maier, M., Williams, S., Bullmore, E., Greenwood, K., Fukuda, R., Ron, M., and Toone, B. (2001). Structural abnormalities in frontal, temporal, and limbic regions and interconnecting white matter tracts in schizophrenic patients with prominent negative symptoms. Am. J. Psychiatry 158, 234-243.

Stein, E. A. (2001). fMRI: a new tool for the in vivo localization of drug actions in the brain. J. Anal. Toxicol. 25, 419-424.

Szendi, I., Kiss, M., Racsmany, M., Boda, K., Cimmer, C., Voros, E., Kovacs, Z. A., Szekeres, G. Galsi, G., Pleh, C., Csernay, L., and Janka, Z. (2006). Correlations between clinical symptoms, working memory functions and structural brain abnormalities in men with schizophrenia. Psychiatry Res. 147, 47-55.

Turetsky, B., Cowell, P. E., Gur, R. C., Grossman, R. I., Shtasel, D. L., and Gur, R. E. (1995). Frontal and temporal lobe brain volumes in schizophrenia. Relationship to symptoms and clinical subtype. Arch. Gen. Psychiatry 52, 1061-1070.

Turkington, D., Sensky, T., Scott, J., Barnes, T. R., Nur, U., Siddle, R., Hammond, K., Samarasekara, N., and Kingdon, D. (2008). A randomized controlled trial of cognitivebehavior therapy for persistent symptoms in schizophrenia: a fiveyear follow-up. Schizophr. Res. 98, $1-7$.

Tzourio-Mazoyer, N., Landeau, B., Papathanassiou, D., Crivello, F., Etard, O., Delcroix, N., Mazoyer, B. and Joliot, M. (2002). Automated anatomical labeling of activations in SPM using a macroscopic anatomical parcellation of the MNI MRI single-subject brain. Neuroimage 15 , 273-289.

Wechsler, D. (1997). Wechsler Adult Intelligence Scale, 3rd Edn. San Antonio, TX: The Psychological Corporation.

Williams, G. V., and Castner, S. A. (2008). Dissecting the biology of prefrontal cortical dysfunction in schizophrenia: deficiency in mnemonic processing. Biol. Psychiatry 64, 1024-1025.

Woods, S. W. (2003). Chlorpromazine equivalent doses for the newer atypical antipsychotics. J. Clin. Psychiatry 64, 663-667.

Yoshida, T., McCarley, R. W., Nakamura, M., Lee, K., Koo, M. S., Bouix, S., Salisbury, D. F., Morra, L., Shenton, M. E., and Niznikiewicz, M. A. (2009).
A prospective longitudinal volumetric MRI study of superior temporal gyrus gray matter and amygdalahippocampal complex in chronic schizophrenia. Schizophr. Res. 113, 84-94.

Zald, D., and Kim, S. (2001). "The orbitofrontal cortex," in The Frontal Lobes and Neuropsychiatric Illness, 1 st Edn, eds S. Salloway, P. Malloy, and J. Duffy (Washington, DC: American Psychiatric Publishing, Inc.), 33-69.

Conflict of Interest Statement: The work was funded by operating grants from CIHR (\#68961) and the Sackler Foundation to Drs. Lepage and A. K. Malla. M. Lepage is supported by a salary award from the FRSQ. A. K. Malla is supported by the Canada Research Chairs program. The CIHR and Sackler Foundation had no further role in study design; in the collection, analysis, and interpretation of data; in the writing of the report; and in the decision to submit the paper for publication. M. Lepage reports having received honorariums for educational events from JanssenOrtho and Lilly during the past 3 years. A. K. Malla reports having received financial assistance/compensation for research and educational activities from Pfizer, Janssen-Ortho, AstraZeneca, and Bristol-Myers-Squibb during the past 3 years. R. Joober reports having received consultancy honorarium from Pfizer and Janssen-Ortho during the past 3 years. Audrey Benoit and Michael Bodnar declare they have no conflicts of interest. There are no other personal financial holdings that could be perceived as constituting a potential conflict of interest.

Received: 21 February 2012; accepted: 19 April 2012; published online: 08 May 2012.

Citation: Benoit A, Bodnar M, Malla AK, Joober R and Lepage M (2012) The structural neural substrates of persistent negative symptoms in first-episode of nonaffective psychosis: a voxel-based morphometry study. Front. Psychiatry 3:42. doi: 10.3389/fpsyt.2012.00042

This article was submitted to Frontiers in Frontiers in Neuropsychiatric Imaging and Stimulation, a specialty of Frontiers in Psychiatry.

Copyright (C) 2012 Benoit, Bodnar, Malla, Joober and Lepage. This is an open-access article distributed under the terms of the Creative Commons Attribution Non Commercial License, which permits non-commercial use, distribution, and reproduction in other forums, provided the original authors and source are credited. 